\title{
Prevalence and Risk Factors of Postpartum Depression in Yaounde, Cameroon
}

\author{
Nadège Djoda Adama*, Pascal Foumane, Jean Pierre Kamga Olen, Julius Sama Dohbit, \\ Esther Ngo Um Meka, Emile Mboudou \\ Faculty of Medicine and Biomedical Sciences, The University of Yaoundé, Yaoundé, Cameroon \\ Email: ${ }^{*}$ nadege.djoda@yahoo.fr
}

Received 13 August 2015; accepted 18 September 2015; published 21 September 2015

Copyright (C) 2015 by authors and Scientific Research Publishing Inc.

This work is licensed under the Creative Commons Attribution International License (CC BY).

http://creativecommons.org/licenses/by/4.0/

c) () Open Access

\begin{abstract}
Introduction: Postpartum Depression is one of the commonest complications of the postpartum period. In Cameroon, little is known about this condition. Our objective was to determine the prevalence and identify the risk factors for postpartum depression. Methodology: The study was carried out at the Yaoundé Gyneco-Obstetric and Pediatric Hospital, from November 4th 2013 to April 4th 2014. All the women between the 4th and 6th week after birth who gave their consent were included. A pretested questionnaire including demographic, psychosocial, maternal and infant variables as well as the Edinburgh Postnatal Depression Scale (EPDS) was filled. A woman with an EPDS score $\geq 12$ was considered having postpartum depression, while a score $<12$ ruled out a postpartum depression. Results: We recruited 214 women, among whom 50 had an EPDS score $\geq$ 12, giving a prevalence of $23.4 \%$ of postpartum depression. After multivariate analysis, the risk factors of postpartum depression were: lack of satisfaction in the marital relationship, recent financial problems, recent conflicts with the partner, baby blues, difficulties in feeding the baby and problems with the baby's sleep. Conclusion: Postpartum depression is common and associated to specific risk factors in our setting.
\end{abstract}

\section{Keywords}

Postpartum Depression, Prevalence, Risk Factors, Cameroon

\section{Introduction}

Becoming a mother is associated with emotional distress in about 30\% of women [1]-[2]. The postpartum period is of particular vulnerability for decompensations or the outbreak of some psychiatric disorders [3]. Postpartum

${ }^{*}$ Corresponding author.

How to cite this paper: Djoda Adama, N., Foumane, P., Kamga Olen, J.P., Sama Dohbit, J., Ngo Um Meka, E. and Mboudou, E. (2015) Prevalence and Risk Factors of Postpartum Depression in Yaounde, Cameroon. Open Journal of Obstetrics and Gynecology, 5, 608-617. http://dx.doi.org/10.4236/ojog.2015.511086 
depression (PPD) is one of the most common complications in women in the postpartum period [4]. Its prevalence is estimated between $10 \%$ and $20 \%$, with an average prevalence of $13 \%$ [5]. This figure depends on the geographic location and the socio-economic conditions. In sub-Saharan Africa, studies have reported a prevalence of $6.6 \%$ in Uganda, $14.6 \%$ in Nigeria, $34.7 \%$ in South Africa, and up to $50.8 \%$ in the Democratic Republic of Congo [6]-[9].

The diagnosis is difficult because of the variability in clinical presentation. In addition, some health professionals do not have the required competence for diagnosis. Therefore, postpartum depression is often confused with baby blues or postpartum psychosis.

The causes are not well known. Some authors have identified several disposing factors to the disease. The most encountered are: a personal history of depression or other psychiatric condition, family history of psychiatric disorder, anxiety and depression during pregnancy, the baby blues, the stress due to the infant, to binding life events, to marital relationship or insufficient social support [10] [11].

The occurrence of postpartum depression can have serious consequences, not only on the mother but also on the mother-infant dyad and indirectly hinder the psycho affective development of the child; hence the importance of early detection. The Edinburgh Postnatal Depression Scale (EPDS) [12] is an internationally recognized tool used for the screening of PPD. This self-administered questionnaire is simple and quick to use. Its acceptability by patients themselves makes it a tool of choice. It consists of 10 multiple choice items rated each on a scale of $0-3$, giving a total score ranging from 0 to 30 [13].

To the best of our knowledge, there are very few available studies on postpartum depression in Cameroon. Thus, the present study was conducted with the general objective to determine its prevalence and identify the risk factors in our environment.

\section{Methodology}

It was an analytical case-control study, carried out at the Yaoundé Gyneco-Obstetrics and Pediatric Hospital and (YGOPH), which is a referral hospital dedicated to the mother and child health. The study period covered 5 months; from November 4th 2013 to April 4th 2014. All women who presented at the postpartum consultation, vaccination or routine pediatric consultation with a child aged 4 to 6 weeks were included. The recruited women had given birth to a live or dead baby at a term $\geq 28$ weeks. They should not be under treatment for a given psychiatric disorder. The sampling was consecutive and based on a calculated minimum sample size of 48 women per group.

Data was collected using a pretested questionnaire with two sections. The first section included sociodemographic characteristics, the relationship with the partner (satisfaction and recent conflicts within the last 3 month), social support from the father of the child, family and friends, stressful life events in the 3 months preceding the birth, personal and family history of psychiatric disorders, obstetric history (parity, history of complications during pregnancy or during delivery or postpartum complications), the experience of the conception, pregnancy and childbirth and the relationship with the newborn. These factors were assessed by yes or no type questions. The second part consisting of the Edimburg Postpartum Depression Scale (EPDS) proposed by Cox et al. in 1987, and better used between the 4th and 6th week postpartum [12].

Before inclusion, women received oral and written information about the study. The coded questionnaire was filled by the patient after reading and signing the consent form. For those who could not read nor write; the questionnaire was administered by a female member of the research team. A woman with an EPDS score $\geq 12$ was considered having postpartum depression, while a score $<12$ ruled out a postpartum depression. All the women with a score $\geq 12$ were referred to a psychiatric center for a specialized care.

Data were analyzed using Chi-square and Fisher's exact test. Risk factors were identified by calculating the odds ratio (OR) with a 95\% confidence interval (CI). P-values less than 0.05 indicate statistical significance. We then made several logistic regressions to eliminate confounding factors.

This study was approved by the Institutional Ethics Committee of Research for Human Health of the Yaounde Gyneco-Obstetrics and Pediatric Hospital.

\section{Results}

Two hundreds and fourteen women were included. The ages ranged from 15 years to 45 years with a mean age of $28.15 \pm 5.22$ years. 


\subsection{Prevalence}

The mean score on the Edinburgh Postnatal Depression Scale was $7.44 \pm 5.65$, with scores ranging from 0 to 26 . Fifty women had a score $\geq 12$ and were considered having postpartum depression, giving a prevalence of $23.4 \%$.

\subsection{Associated Risk Factors}

\subsubsection{Sociodemographic Characteristics}

No socio-demographic variable was found to be a risk factor for postpartum depression, as shown in Table 1.

Table 1. Sociodemographic factors associated with potspartum depression.

\begin{tabular}{|c|c|c|c|c|}
\hline \multirow{3}{*}{ Factors } & \multicolumn{2}{|c|}{ Potspartum depression } & \multirow{3}{*}{$\begin{array}{l}\text { Odds Ratio } \\
\text { (CI 95\%) }\end{array}$} & \multirow{3}{*}{ P value } \\
\hline & Yes $($ EPDS $\geq 12)$ & No $($ EPDS < 12) & & \\
\hline & Effectif (\%) & Effectif (\%) & & \\
\hline \multicolumn{5}{|l|}{ Age } \\
\hline 15 - 19 years & $3(6)$ & $6(3.7)$ & $1.68(0.40-6.98)$ & 0.35 \\
\hline 20 - 29 years & $31(62)$ & $98(59.8)$ & $1.90(0.57-2.11)$ & 0.45 \\
\hline 30 - 39 years & $15(30)$ & $56(34.1)$ & $0.83(0.42-1.64)$ & 0.35 \\
\hline$\geq 40$ years & $1(2)$ & $4(2.4)$ & $0.82(0.09-7.47)$ & 0.67 \\
\hline \multicolumn{5}{|l|}{ Cultural area } \\
\hline Sahel & $4(2)$ & $9(5.5)$ & $0.35(0.03-2.84)$ & 0.28 \\
\hline Forest & $20(40)$ & 49 (29.9) & $1.56(0.81-3.02)$ & 0.09 \\
\hline Coast & $4(8)$ & $9(5.5)$ & $1.50(0.44-5.09)$ & 0.36 \\
\hline Grass field & $25(50)$ & $96(58.5)$ & $0.71(0.38-1.34)$ & 0.15 \\
\hline Non Cameroonian & $0(0)$ & $1(0.6)$ & - & 0.77 \\
\hline \multicolumn{5}{|l|}{ Religion } \\
\hline Catholic & $29(58)$ & $105(64)$ & $0.77(0.40-1.48)$ & 0.27 \\
\hline Protestant & $12(24)$ & 35 (21.3) & $1.16(0.55-2.46)$ & 0.41 \\
\hline Muslim & $1(2)$ & $8(4.9)$ & $0.40(0.05-3.26)$ & 0.34 \\
\hline Other & $8(16)$ & $16(9.8)$ & $1.76(0.70-3.26)$ & 0.16 \\
\hline \multicolumn{5}{|l|}{ Profession } \\
\hline Housewife & $9(18)$ & $42(25.6)$ & $0.64(0.28-1.42)$ & 0.18 \\
\hline Pupil/Student & $8(16)$ & 26 (15.9) & $1.01(0.42-2.40)$ & 0.57 \\
\hline Public sector & $8(16)$ & $25(15.2)$ & $1.06(0.44-2.52)$ & 0.53 \\
\hline Private sector & $10(20)$ & 32 (19.5) & $1.03(0.47-2.28)$ & 0.54 \\
\hline Peasant & $0(0)$ & $1(0.6)$ & - & 0.77 \\
\hline Shopping & $2(4)$ & $10(6.1)$ & $0.64(0.14-3.03)$ & 0.44 \\
\hline Unemployed & $10(10)$ & 19 (11.6) & $1.90(0.22-4.43)$ & 0.10 \\
\hline Small independent & $3(6)$ & $9(5.5)$ & $1.10(0.28-4.22)$ & 0.56 \\
\hline \multicolumn{5}{|l|}{ Marital status } \\
\hline Single & $20(40)$ & $43(26.2)$ & $1.88(0.97-3.64)$ & 0.04 \\
\hline Divorced/separated & $0(0)$ & $3(1.8)$ & - & 0.45 \\
\hline Married/Cohabitating & $30(60)$ & $118(72)$ & $0.58(0.30-1.13)$ & 0.08 \\
\hline \multicolumn{5}{|l|}{ Type of marriage } \\
\hline Monogamy & $28(93.3)$ & 101 (85.6) & $2.35(0.51-10.81)$ & 0.21 \\
\hline Polygamy & $2(6.7)$ & $17(14.4)$ & $0.42(0.09-1.95)$ & 0.21 \\
\hline \multicolumn{5}{|l|}{ Study level } \\
\hline None & $0(0)$ & $1(0.6)$ & - & 0.76 \\
\hline Primary & $3(6)$ & $7(4.3)$ & $1.43(0.36-5.75)$ & 0.42 \\
\hline Secondary & $20(40)$ & 54 (32.9) & $1.36(0.71-2.61)$ & 0.22 \\
\hline University & $27(54)$ & $102(62.2)$ & $0.71(0.38-1.35)$ & 0.19 \\
\hline
\end{tabular}




\subsubsection{Psychosocial Factors}

The psychosocial factors found to be risk factors associated with postpartum depression were: lack of satisfaction in the marital relationship (for married or cohabiting women), recent conflicts with the partner, recent financial problems and loss of employment (Table 2).

The lack of the father's of the child support was the basis of many risk factors identified at univariate analysis: inadequate emotional or financial support, inability to rely or confide on him. The absence of family emotional support was also a risk factor resulting from analysis (Table 3). In the past history, abortion was significantly associated to postpartum depression (Table 4).

\subsubsection{Obstetric Factors}

The conditions in relationship with the last pregnancy associated with postpartum depression were: an unplanned pregnancy, an unwanted pregnancy, depressive symptoms during pregnancy and anxiety during pregnancy (Table 5 and Table 6).

\subsubsection{Neonatal and Postnatal Factors}

As shown in Table 7, having difficulties in feeding the baby and having trouble with his sleep were significantly associated with postpartum depression. At the same time, women who thought they had the baby blues were more likely to experience postpartum depression.

\subsubsection{Factors Associated with Postpartum Depression after Logistic Regression}

After logistic regression, lack of marital satisfaction, recent conflicts either with partner or child's father, recent financial problems, baby blues, having difficulty feeding the baby and having trouble with the baby's sleep remained significant factors associated with postpartum depression.

\section{Discussion}

\subsection{Prevalence of Postpartum Depression}

The prevalence of postpartum depression in our study was $23.4 \%$. This figure is similar to the $23 \%$ of prevalence found in Nigeria by Owoeye et al. [14]. This can be explained by the fact that postpartum depression was

Table 2. Stressful life events associated with potspartum depression.

\begin{tabular}{|c|c|c|c|c|c|}
\hline \multirow{3}{*}{ Factors } & & \multicolumn{2}{|c|}{ Postpartum depression } & \multirow{3}{*}{$\begin{array}{l}\text { Odds Ratio } \\
\text { (CI 95\%) }\end{array}$} & \multirow{3}{*}{$P$ value } \\
\hline & & \multirow{2}{*}{$\begin{array}{l}\text { Yes (EPDS } \geq 12) \\
\text { Effecti } f(\%)\end{array}$} & \multirow{2}{*}{$\begin{array}{l}\text { No (EPDS < 12) } \\
\text { Effectif (\%) }\end{array}$} & & \\
\hline & & & & & \\
\hline \multirow{2}{*}{ Marital satisfaction } & Yes & $18(60)$ & $115(97.5)$ & $0.04(0.01-0.15)$ & 0.00 \\
\hline & No & $12(40)$ & $3(2.5)$ & $\underline{25.56(6.56-99.49)}$ & $\underline{0.00}$ \\
\hline \multirow{2}{*}{ Recent conflicts with the partner } & $\underline{\text { Yes }}$ & $31(62)$ & 39 (23.8) & $\underline{5.23(2.66-10.27)}$ & $\underline{0.00}$ \\
\hline & No & $19(38)$ & $125(76.2)$ & $0.19(0.10-0.38)$ & 0.00 \\
\hline \multirow{2}{*}{ Financial problems } & $\underline{\text { Yes }}$ & $43(86)$ & $86(52.4)$ & $\underline{5.57(2.36-13.11)}$ & $\underline{0.00}$ \\
\hline & No & $7(14)$ & $78(47.6)$ & $0.18(0.08-0.42)$ & 0.00 \\
\hline \multirow{2}{*}{ Conflicts with the family } & Yes & $18(36)$ & 37 (22.6) & $1.93(0.97-3.92)$ & 0.04 \\
\hline & No & $32(64)$ & $127(77.4)$ & $0.52(0.26-1.03)$ & 0.04 \\
\hline \multirow{2}{*}{ Loss of a love one } & Yes & $17(34)$ & 49 (29.9) & $1.20(0.61-2.37)$ & 0.35 \\
\hline & No & $33(66)$ & $115(70.1)$ & $0.83(0.42-1.62)$ & 0.35 \\
\hline \multirow{2}{*}{ Serious illness in the family } & Yes & $16(32)$ & 39 (23.8) & $1.50(0.75-3.02)$ & 0.16 \\
\hline & No & $34(68)$ & 125 (76.2) & $0.66(0.33-1.33)$ & 0.16 \\
\hline \multirow{2}{*}{ Job loss } & Yes & $10(20)$ & $15(9.3)$ & $2.45(1.02-5.87)$ & 0.04 \\
\hline & No & $40(80)$ & 149 (90.7) & $0.40(0.17-0.96)$ & 0.04 \\
\hline
\end{tabular}


Table 3. Social support and postpartum depression.

\begin{tabular}{|c|c|c|c|c|c|}
\hline \multirow{3}{*}{ Factors } & & \multicolumn{2}{|c|}{ Postpartum depression } & \multirow{3}{*}{$\begin{array}{l}\text { Odds Ratio } \\
\text { (CI 95\%) }\end{array}$} & \multirow{3}{*}{ P value } \\
\hline & & \multirow{2}{*}{$\begin{array}{l}\text { Yes (EPDS } \geq 12) \\
\%\end{array}$} & \multirow{2}{*}{$\begin{array}{l}\text { No }(\text { EPDS < 12) } \\
\%\end{array}$} & & \\
\hline & & & & & \\
\hline \multicolumn{6}{|c|}{ Child's father support } \\
\hline \multirow{2}{*}{ Adequate emotional support } & Yes & $32(64)$ & $138(84.1)$ & $0.33(0.16-0.68)$ & 0.00 \\
\hline & $\underline{\text { No }}$ & $18(36)$ & $26(15.9)$ & $\underline{2.98(1.46-6.09)}$ & $\underline{0.00}$ \\
\hline \multirow{2}{*}{ Adequate instrumental support } & Yes & $33(66)$ & 146 (89) & $0.24(0.11-0.51)$ & 0.00 \\
\hline & $\underline{\text { No }}$ & $17(44)$ & $18(11)$ & $\underline{4.18(1.95-8.96)}$ & $\underline{0.00}$ \\
\hline \multirow{2}{*}{ Can rely on him } & Yes & $32(64)$ & $147(89.6)$ & $0.20(0.09-0.44)$ & 0.00 \\
\hline & $\underline{\text { No }}$ & $18(36)$ & $19(10.4)$ & $4.86(2.26-10.45)$ & $\underline{0.00}$ \\
\hline \multirow{2}{*}{ Can confide on him } & Yes & $30(58.8)$ & $135(82.8)$ & $0.30(0.15-0.59)$ & 0.00 \\
\hline & $\underline{\text { No }}$ & $21(41.2)$ & $28(17.2)$ & $\underline{3.38(1.69-6.73)}$ & $\underline{0.00}$ \\
\hline \multicolumn{6}{|c|}{ Family support } \\
\hline \multirow{2}{*}{ Adequate emotional support } & Yes & $39(78)$ & $147(89.6)$ & $0.41(0.18-0.95)$ & 0.03 \\
\hline & $\underline{\text { No }}$ & $11(22)$ & $107(10.4)$ & $\underline{2.44(1.06-5.63)}$ & $\underline{0.03}$ \\
\hline \multirow{2}{*}{ Adequate instrumental support } & Yes & $35(70)$ & 131 (79.9) & $0.59(0.29-1.20)$ & 0.10 \\
\hline & No & $15(30)$ & $33(21.1)$ & $1.70(0.83-3.48)$ & 0.10 \\
\hline \multirow{2}{*}{ Can rely on them } & Yes & $42(84)$ & $148(90.2)$ & $0.57(0.23-1.42)$ & 0.17 \\
\hline & No & $8(16)$ & $16(9.8)$ & $1.76(0.70-4.40)$ & 0.17 \\
\hline \multirow{2}{*}{ Can confide on them } & Yes & $41(82)$ & $147(89.6)$ & $0.53(0.22-1.27)$ & 0.12 \\
\hline & No & $9(18)$ & $17(10.4)$ & $1.90(0.79-4.57)$ & 0.12 \\
\hline \multicolumn{6}{|c|}{ Friends support } \\
\hline \multirow{2}{*}{ Adequate emotional support } & Yes & $40(80)$ & $126(76.8)$ & $1.20(0.55-2.64)$ & 0.40 \\
\hline & No & $10(20)$ & $38(23.2)$ & $2.44(1.05-5.63)$ & 0.40 \\
\hline \multirow{2}{*}{ Adequate instrumental support } & Yes & $27(54)$ & $92(56.1)$ & $0.92(0.49-1.73)$ & 0.50 \\
\hline & No & $23(46)$ & $72(43.9)$ & $1.08(0.58-2.05)$ & 0.50 \\
\hline \multirow{2}{*}{ Can rely on them } & Yes & $29(58)$ & $103(62.8)$ & $0.82(0.43-1.55)$ & 0.33 \\
\hline & No & $21(42)$ & $61(37.2)$ & $1.22(0.64-2.33)$ & 0.33 \\
\hline \multirow{2}{*}{ Can confide on them } & Yes & $21(42)$ & $87(53)$ & $0.64(0.34-1.21)$ & 0.11 \\
\hline & No & $29(58)$ & $77(47)$ & $1.56(0.82-2.96)$ & 0.11 \\
\hline
\end{tabular}

detected by the same tool in a similar setting: Edinburg Postpartum Depression Scale with a threshold score at 12 in women at 6 weeks postpartum in a referral hospital. However; this prevalence is higher than the average prevalence of $13 \%$ described by O'Hara and Swain in the Western [5] or the18.4\% figure of Sawyer et al. in Africa [15]. At the same time, higher figures have been witnessed in some African countries: 43\% in Uganda [1] and 50.8\% in the Democratic Republic of Congo [9]. In these two studies, Edinburg Postpartum Scale was not the tool used to identify women with postpartum depression.

\subsection{Risk Factors Associated with Postpartum Depression}

At multivariate analysis, identified risk factors for postpartum depression were: lack of satisfaction in the marital relationship, recent conflicts with the partner, recent financial problems, difficulties in feeding the baby, problems with the baby's sleep and baby blues. 
Table 4. Past history and postpartum depression.

\begin{tabular}{|c|c|c|c|c|}
\hline \multirow{3}{*}{ Factors } & \multicolumn{2}{|c|}{ Postpartum depression } & \multirow{3}{*}{$\begin{array}{l}\text { Odds Ratio } \\
\text { (CI 95\%) }\end{array}$} & \multirow{3}{*}{$P$ value } \\
\hline & Yes (EPDS $\geq 12)$ & No $($ EPDS $<12)$ & & \\
\hline & $\%$ & $\%$ & & \\
\hline \multicolumn{5}{|l|}{ Parity } \\
\hline - 1 & $18(36)$ & $99(36)$ & $1(0.52-1.94)$ & 0.56 \\
\hline - 2 and more & $32(64)$ & 105 (64) & $1(0.52-1.93)$ & 0.56 \\
\hline \multicolumn{5}{|l|}{ Abortion history } \\
\hline - Yes & $13(26)$ & $16(9.8)$ & $\underline{3.25(1.44-7.35)}$ & $\underline{0.00}$ \\
\hline - No & $37(74)$ & $148(90.2)$ & $0.31(0.14-0.69)$ & 0.00 \\
\hline \multicolumn{5}{|c|}{ Number of living children } \\
\hline - More than 4 & $3(6)$ & $17(10.4)$ & $0.55(0.15-1.97)$ & 0.27 \\
\hline - 1 - 3 & $47(94)$ & $147(89.6)$ & $1.18(0.51-6.45)$ & 0.27 \\
\hline \multicolumn{5}{|c|}{ Complications and previous pregnancies } \\
\hline \multicolumn{5}{|l|}{ During a pregnancy } \\
\hline - Yes & $11(34.4)$ & $22(21)$ & $1.98(0.83-4.71)$ & 0.09 \\
\hline - No & $19(65.5)$ & $85(79)$ & $0.55(0.24-1.23)$ & 0.09 \\
\hline \multicolumn{5}{|l|}{ During a childbirth } \\
\hline - Yes & $5(17.9)$ & $16(16.3)$ & $1.11(0.37-3.36)$ & 0.52 \\
\hline - No & $23(82.1)$ & $82(83.7)$ & $0.97(0.34-2.80)$ & 0.52 \\
\hline \multicolumn{5}{|c|}{ During postpartum period } \\
\hline - Yes & $3(10.7)$ & $2(2)$ & $5.76(0.91-36.36)$ & 0.07 \\
\hline - No & $23(89.3)$ & $96(98)$ & $0.19(0.03-1.19)$ & 0.07 \\
\hline \multicolumn{5}{|l|}{ Baby health problem } \\
\hline - Yes & 5 (17.9) & $17(17.3)$ & $1.03(0.34-3.11)$ & 0.57 \\
\hline - No & $23(82.1)$ & 81 (82.7) & $1.04(0.36-2.98)$ & 0.57 \\
\hline
\end{tabular}

The lack of satisfaction in the marital relationship is a well documented risk factor for postpartum depression. Authors from Africa, Asia and Europe have stated a significant association this factor and postpartum depression [16]-[19]. At the contrary, an Iranian study failed to reach a similar conclusion [20].

As far as pregnancy and childbirth are concerned, the marital partner plays a major role. Therefore, a conflict with the partner is an obvious risk factor for postpartum depression. This has already been objected by Husain et al. in Pakistan [21].

Recent financial problems are independent risk factors found in our study. This has been published as risk factor for postpartum depression by Amr et al. in Saudi Arabia [17]. The same authors also identified loss of employment as a risk factor.

Difficulty in feeding the baby is a stressful condition for the mother and significantly leads to postpartum depression, according to our findings. For Kakyo et al. in Uganda [1], high levels of postpartum depression are significantly associated with having a child which is not feeding well. This association can be explained by the stress due the fear of malnourishment for the baby.

As well as difficulties in feeding the baby, problems with the baby's sleep have been were found to be a risk factor for postpartum depression in our study. This can be understood by the fact that any disturbance of the baby sleep's lead to the absence of sleeping for the mother, which is a stressful condition. 
Table 5. Obstetric factors associated with postpartum depression.

\begin{tabular}{|c|c|c|c|c|}
\hline \multirow{3}{*}{ Factors } & \multicolumn{2}{|c|}{ Postpartum depression } & \multirow{3}{*}{$\begin{array}{l}\text { Odds Ratio } \\
\text { (CI 95\%) }\end{array}$} & \multirow{3}{*}{$P$ value } \\
\hline & Yes (EPDS $\geq 12)$ & No (EPDS < 12) & & \\
\hline & $\%$ & $\%$ & & \\
\hline \multicolumn{5}{|l|}{ Planned pregnancy } \\
\hline - Yes & $23(46)$ & $116(70.7)$ & $0.35(0.18-0.67)$ & 0.00 \\
\hline • No & $27(54)$ & $48(29.3)$ & $\underline{2.82(1.48-5.43)}$ & $\underline{0.00}$ \\
\hline \multicolumn{5}{|l|}{ Conception } \\
\hline • Natural & $49(98)$ & $163(99.4)$ & $0.30(0.02-4.90)$ & 0.41 \\
\hline - In vitro fertilization & $1(2)$ & $1(0.6)$ & $3.32(0.20-54.17)$ & 0.41 \\
\hline \multicolumn{5}{|l|}{ Desired pregnancy } \\
\hline • Yes & $39(78)$ & $153(93.3)$ & $0.25(0.10-0.53)$ & 0.00 \\
\hline - $\underline{\text { No }}$ & $11(22)$ & $11(6.3)$ & $\underline{3.92(1.58-9.71)}$ & $\underline{0.00}$ \\
\hline \multicolumn{5}{|l|}{ Number of antenatal cares } \\
\hline - Less than 4 & $16(32)$ & $43(26.4)$ & $1.28(0.64-2.55)$ & 0.29 \\
\hline - 4 and more & $34(68)$ & $120(73.6)$ & $0.75(0.39-1.55)$ & 0.29 \\
\hline \multicolumn{5}{|l|}{ Full antenatal diagnosis } \\
\hline - Yes & $43(86)$ & $146(89.6)$ & $0.71(0.28-1.84)$ & 0.32 \\
\hline - No & $7(14)$ & $17(10.4)$ & $1.32(0.52-3.37)$ & 0.32 \\
\hline \multicolumn{5}{|c|}{ Depressive symptoms during pregnancy } \\
\hline - $\underline{\text { Yes }}$ & $24(48)$ & $26(15.9)$ & $\underline{4.90(2.44-9.82)}$ & $\underline{0.00}$ \\
\hline - No & $26(52)$ & $138(84.1)$ & $0.20(0.10-0.41)$ & 0.00 \\
\hline \multicolumn{5}{|l|}{ Anxiety during pregnancy } \\
\hline - Yes & $35(70)$ & $75(45.7)$ & $\underline{2.77(1.40-5.46)}$ & $\underline{0.00}$ \\
\hline - No & $14(30)$ & $90(54.3)$ & $0.36(0.18-0.71)$ & 0.00 \\
\hline \multicolumn{5}{|l|}{ Disease during pregnancy } \\
\hline - Yes & $20(40)$ & $47(28.7)$ & $1.66(0.85-3.20)$ & 0.09 \\
\hline - No & $30(60)$ & $117(71.3)$ & $0.60(0.31-1.16)$ & 0.09 \\
\hline \multicolumn{5}{|l|}{ Antepartum complication } \\
\hline - Yes & $10(20)$ & $23(14)$ & $1.53(0.67-3.48)$ & 0.20 \\
\hline - No & $40(80)$ & $141(86)$ & $0.65(0.29-1.48)$ & 0.20 \\
\hline \multicolumn{5}{|l|}{ Delivery } \\
\hline - Premature & $6(12)$ & $22(13.4)$ & $0.88(0.33-2.31)$ & 0.50 \\
\hline - At term & $44(88)$ & $142(86.6)$ & $1.14(0.43-2.98)$ & 0.50 \\
\hline \multicolumn{5}{|l|}{ Type of delivery } \\
\hline • Normal & $41(82)$ & $136(82.9)$ & $0.94(0.41-2.15)$ & 0.51 \\
\hline - Cesarean section & $8(18)$ & $29(17.1)$ & $1.07(0.46-2.44)$ & 0.51 \\
\hline \multicolumn{5}{|l|}{ Delivery } \\
\hline - Spontaneous & $35(85.4)$ & $113(83.1)$ & $1.19(0.45-3.15)$ & 0.47 \\
\hline • Induced & $6(14.6)$ & $23(16.9)$ & $0.95(0.48-1.89)$ & 0.47 \\
\hline \multicolumn{5}{|l|}{ Instrumental delivery } \\
\hline - Yes & $2(4.9)$ & $4(2.9)$ & $1.69(0.30-9.59)$ & 0.42 \\
\hline • No & 39 (95.1) & $132(97.1)$ & $0.60(0.11-3.38)$ & 0.42 \\
\hline \multicolumn{5}{|l|}{ Cesarean section } \\
\hline - Elective & $1(11.1)$ & $8(28.6)$ & $0.31(0.03-2.92)$ & 0.28 \\
\hline • In emergency & $8(88.9)$ & $20(71.4)$ & $2.51(0.31-20.59)$ & 0.28 \\
\hline \multicolumn{5}{|l|}{ Complication in childbirth } \\
\hline - Yes & $3(6)$ & $6(3.7)$ & $1.68(0.40-6.98)$ & 0.35 \\
\hline • No & $47(94)$ & $158(96.3)$ & $0.59(0.14-2.47)$ & 0.35 \\
\hline \multicolumn{5}{|l|}{ Postpartum complication } \\
\hline - Yes & $4(8)$ & $12(7.3)$ & $1.10(0.34-3.58)$ & 0.54 \\
\hline • No & $46(92)$ & $152(92.7)$ & $0.91(0.28-2.95)$ & 0.54 \\
\hline
\end{tabular}


Table 6. neonatal and postnatal variables associated with postpartum depression.

\begin{tabular}{|c|c|c|c|c|}
\hline \multirow{3}{*}{ Factors } & \multicolumn{2}{|c|}{ Postpartum depression } & \multirow{3}{*}{$\begin{array}{l}\text { Odds Ratio } \\
\text { (CI 95\%) }\end{array}$} & \multirow{3}{*}{$P$ value } \\
\hline & Yes (EPDS $\geq 12$ ) & No (EPDS < 12) & & \\
\hline & $\%$ & $\%$ & & \\
\hline \multicolumn{5}{|l|}{ Number of fetuses } \\
\hline - 1 & $46(92)$ & $158(96.3)$ & $0.44(0.11-1.61)$ & 0.18 \\
\hline - 2 and more & $4(8)$ & $6(3.7)$ & $2.29(0.12-8.46)$ & 0.18 \\
\hline \multicolumn{5}{|l|}{ Sex } \\
\hline - Male & $30(55.6)$ & $80(47.1)$ & $1.41(0.75-2.60)$ & 0.17 \\
\hline - Female & $24(44.4)$ & $90(52.9)$ & $0.71(0.38-1.32)$ & 0.17 \\
\hline \multicolumn{5}{|c|}{ Immediate cry at birth } \\
\hline - Yes & $47(37)$ & $148(87.1)$ & $1(0.40-2.48)$ & 0.58 \\
\hline • No & $7(13)$ & $22(12.9)$ & $1(0.40-2.49)$ & 0.58 \\
\hline \multicolumn{5}{|c|}{ Baby reanimated at birth } \\
\hline - Yes & $8(14.8)$ & $17(10)$ & $1.56(0.63-3.86)$ & 0.23 \\
\hline • No & $46(85.2)$ & $153(90)$ & $0.64(0.26-1.58)$ & 0.23 \\
\hline \multicolumn{5}{|c|}{ Health problem at birth } \\
\hline - Yes & $9(16.7)$ & $32(18.8)$ & $0.86(0.38-1.94)$ & 0.44 \\
\hline • No & $45(83.3)$ & $138(81.2)$ & $1.16(0.51-2.61)$ & 0.44 \\
\hline \multicolumn{5}{|c|}{ Difficulties to feed the baby } \\
\hline - Yes & $9(16.7)$ & $8(4.7)$ & $\underline{4.05(1.48-11.10)}$ & $\underline{0.00}$ \\
\hline - No & $45(83.3)$ & $162(95.3)$ & $0.25(0.09-0.68)$ & 0.00 \\
\hline \multicolumn{5}{|c|}{ Problem with the baby's sleep } \\
\hline - $\underline{\text { Yes }}$ & $27(50)$ & $53(31.2)$ & $\underline{2.21(1.18-4.12)}$ & $\underline{0.00}$ \\
\hline - No & $27(50)$ & $117(68.8)$ & $0.45(0.24-0.84)$ & 0.00 \\
\hline \multicolumn{5}{|c|}{ Difficulties in carrying the baby } \\
\hline - Yes & $8(14.8)$ & $19(11.2)$ & $1.38(0.57-3.36)$ & 0.31 \\
\hline - No & $42(85.2)$ & $155(88.8)$ & $0.72(0.30-1.76)$ & 0.31 \\
\hline \multicolumn{5}{|l|}{ The baby cries a lot } \\
\hline - Yes & $15(27.8)$ & $30(17.6)$ & $1.79(0.89-3.66)$ & 0.08 \\
\hline - No & $39(72.2)$ & $140(82.4)$ & $0.56(0.27-1.14)$ & 0.08 \\
\hline \multicolumn{5}{|c|}{ The baby is difficult to calm } \\
\hline - Yes & $9(16.7)$ & $15(8.8)$ & $2.07(0.85-5.03)$ & 0.09 \\
\hline - No & $45(83.3)$ & $155(91.2)$ & $0.48(1.20-1.18)$ & 0.09 \\
\hline \multicolumn{5}{|l|}{ breastfeeding } \\
\hline - Maternal & 21 (38.9) & 77 (45.3) & $0.77(0.41-1.43)$ & 0.25 \\
\hline - Artificial & $2(3.7)$ & $7(4.1)$ & $0.89(0.20-5.08)$ & 0.63 \\
\hline - Both & $31(54.4)$ & $86(50.6)$ & $1.32(0.71-2.44)$ & 0.24 \\
\hline \multicolumn{5}{|l|}{ Baby blues } \\
\hline - Yes & $31(62)$ & $48(29.3)$ & $\underline{3.94(2.03-7.65)}$ & $\underline{0.00}$ \\
\hline - No & 49 (38) & $116(70.7)$ & $0.25(0.13-0.49)$ & 0.00 \\
\hline
\end{tabular}


Table 7. Factors significantly associated with postpartum depression after multiple logistic regressions.

\begin{tabular}{|c|c|c|c|}
\hline Factors & Adjusted Odds Ratio & CI (95\%) & P Value \\
\hline lack of satisfaction in the marital relationship & $\underline{6.91}$ & $\underline{3.29-14.49}$ & $\underline{\mathbf{0 . 0 0}}$ \\
\hline Recent conflicts with the partner & $\underline{2.55}$ & $\underline{1.05-6.18}$ & $\underline{0.04}$ \\
\hline Lack of adequate emotional support of the child's father & 1.32 & $0.45-3.93$ & 0.61 \\
\hline Lack of adequate material support the father of the child & 1.28 & $0.30-5.43$ & 0.74 \\
\hline Cannot rely on the father of the child & 3.10 & $0.76-12.64$ & 0.11 \\
\hline Can trust the father of the child & 1.18 & $0.48-2.91$ & 0.72 \\
\hline Lack of emotional family support & 2.24 & $0.79-6.35$ & 0.13 \\
\hline$\underline{\text { Recent financial problems }}$ & $\underline{3.85}$ & $\underline{1.44-10.28}$ & $\underline{0.01}$ \\
\hline Loss of a job recently & 1.93 & $0.64-5.78$ & 0.24 \\
\hline Abortion history & 2.73 & $0.95-7.81$ & 0.06 \\
\hline Unplanned pregnancy & 1.08 & $0.27-4.35$ & 0.91 \\
\hline Unwanted pregnancy & 1.64 & $0.66-4.12$ & 0.29 \\
\hline Depressive symptoms during pregnancy & 1.66 & $0.71-3.89$ & 0.24 \\
\hline Anxiety during pregnancy & 1.36 & $0.56-3.31$ & 0.49 \\
\hline Baby blues & $\underline{3.52}$ & $\underline{1.48-8.41}$ & $\underline{\mathbf{0 . 0 0}}$ \\
\hline Difficulties in feeding the baby & $\underline{3.51}$ & $\underline{1.26-9.82}$ & $\underline{0.02}$ \\
\hline Problems with the baby's sleep & $\underline{2.02}$ & $\underline{1.06-3.81}$ & $\underline{0.03}$ \\
\hline
\end{tabular}

Signs of baby blues described by the mother after birth were independently associated with postpartum depression. Many studies have documented the same finding. For example, Lee et al. have stated baby blues as a risk factor for postpartum depression in China [22].

Our results should be considered with some limitations, as women might not give the correct answers to the questions. In addition, the Edinburg Postpartum Depression Scale is a screening tool that shows depressive symptoms in the last 7 days, not an instrument for generating a diagnosis of depression. All women with a score $\geq 12$ were oriented to a psychiatrist, but only few of them met him. All these might have introduced bias in this study.

\section{Conclusion}

This study stated the prevalence of postpartum depression in our setting at $23.4 \%$. Independent risk factors associated with postpartum depression are: lack of satisfaction in the marital relationship, recent conflicts with the partner/father of the child, recent financial problems, baby blues, difficulties in feeding the baby and problems with the baby's sleeping. The routine screening and management of postpartum depression in primary health care are recommended, so as to improve maternal and child wellbeing.

\section{References}

[1] Kakyo, T.A., Muliira, J.K., Mbalinda, S.N., Kizza, I.B. and Muliira, R.S. (2012) Factors Associated with Depressive Symptoms among Postpartum Mothers in a Rural District in Uganda. Midwifery, 28, 374-379. http://dx.doi.org/10.1016/j.midw.2011.05.001

[2] Bener, A., Gerber, L.M. and Sheikh, J. (2012) Prevalence of Psychiatric Disorders and Associated Risk Factors in Women during Their Postpartum Period: A Major Public Health Problem and Global Comparison. International Journal of Women's Health, 4, 191-200. http://dx.doi.org/10.2147/IJWH.S29380

[3] Masmoudi, J., Trabelsi, S., Charfeddine, F. and Jaoua, A. (2006) La dépression du post partum. JIM SFAX, 11-12, 7-13.

[4] Motzfeldt, I., Andreasen, S., Pedersen, A.L. and Pedersen, M.L. (2013) Prevalence of Postpartum Depression in Nuuk, 
Greenland-A Cross-Sectional Study Using Edinburgh Postnatal Depression Scale. International Journal of Circumpolar Health, 72. http://www.ncbi.nlm.nih.gov/pmc/articles/PMC3753142/

[5] O’hara, M.W. and Swain, A.M. (1996) Rates and Risk of Postpartum Depression-A Meta-Analysis. International Review of Psychiatry, 8, 37-54. http://dx.doi.org/10.3109/09540269609037816

[6] Nakku, J.E., Nakasi, G. and Mirembe, F. (2006) Postpartum Major Depression at Six Weeks in Primary Health Care: Prevalence and Associated Factors. African Health Sciences, 6, 207-14.

[7] Adewuya, A.O., Fatoye, F.O., Ola, B.A., Ijaodola, O.R. and Ibigbami, S.-MO. (2005) Sociodemographic and Obstetric Risk Factors for Postpartum Depressive Symptoms in Nigerian Women. Journal of Psychiatric Practice, 11, 353-358. http://dx.doi.org/10.1097/00131746-200509000-00009

[8] Cooper, P.J., Tomlinson, M., Swartz, L., Woolgar, M., Murray, L. and Molteno, C. (1999) Post-Partum Depression and the Mother-Infant Relationship in a South African Peri-Urban Settlement. British Journal of Psychiatry, 175, 554-558. http://dx.doi.org/10.1192/bjp.175.6.554

[9] Imbula Essam, B., Okitundu Luwa, E-.AD. and Mampunza Ma-Miezi, S. (2012) [Postpartum Depression in Kinshasa (DR Congo): Prevalence and Risk Factors]. Médecine et Santé Tropicales, 22, 379-384.

[10] Robertson, E., Grace, S., Wallington, T. and Stewart, D.E. (2004) Antenatal Risk Factors for Postpartum Depression: A Synthesis of Recent Literature. General Hospital Psychiatry, 26, 289-95. http://dx.doi.org/10.1016/j.genhosppsych.2004.02.006

[11] Stewart, D.E., Robertson, E., Dennis, C.-L., Grace, S.L. and Wallington, T. (2003) Postpartum Depression: Literature Review of Risk Factors and Interventions.

[12] Cox, J.L., Holden, J.M. and Sagovsky, R. (1987) Detection of Postnatal Depression. Development of the 10-Item Edinburgh Postnatal Depression Scale. British Journal of Psychiatry, 150, 782-786. http://dx.doi.org/10.1192/bjp.150.6.782

[13] Jardri, R. (2004) Le dépistage de la dépression postnatale: Revue qualitative des études de validation de l’Edinburgh Postnatal Depression Scale. Devenir, 16, 245-262. http://dx.doi.org/10.3917/dev.044.0245

[14] Owoeye, A.O., Aina, O.F. and Morakinyo, O. (2006) Risk Factors of Postpartum Depression and EPDS Scores in a GROUP of Nigerian women. Tropical Doctor, 36, 100-103. http://dx.doi.org/10.1258/004947506776593341

[15] Sawyer, A., Ayers, S. and Smith, H. (2010) Pre- and Postnatal Psychological Wellbeing in Africa: A Systematic Review. Journal of Affective Disorders, 123, 17-29. http://dx.doi.org/10.1016/j.jad.2009.06.027

[16] Dmitrovic, B.K., Dugalic, M.G., Balkoski, G.N., Dmitrovic, A. and Soldatovic, I. (2013) Frequency of Perinatal Depression in Serbia and Associated Risk Factors. International Journal of Social Psychiatry, 60, 528-532.

[17] Amr, M.A., Balaha, M. and Al Moghannum, M. (2012) Postpartum Mental Health among Young Women. Online Journal of Health and Allied Sciences. http://cogprints.org/8856/

[18] Al Dallal, F.H. and Grant, I.N. (2012) Postnatal Depression among Bahraini Women: Prevalence of Symptoms and Psychosocial Risk Factors. East Mediterr Heal J Rev Santé Méditerranée Orient Al-Majallah Al-Ṣihhịyah Li-Sharq Al-Mutawassit, 18, 439-445.

[19] Meçe, D. (2013) Postpartum Depression and Marital Relationship. Academic Journal of Interdisciplinary Studies http://www.mcser.org/journal/index.php/ajis/article/view/138

[20] Taherifard, P., Delpisheh, A., Shirali, R., Afkhamzadeh, A. and Veisani, Y. (2013) Socioeconomic, Psychiatric and Materiality Determinants and Risk of Postpartum Depression in Border City of Ilam, Western Iran. Depression Research and Treatment. http://www.hindawi.com/journals/drt/2013/653471/abs/

[21] Husain, N., Bevc, I., Husain, M., Chaudhry, I.B., Atif, N. and Rahman, A. (2006) Prevalence and Social Correlates of Postnatal Depression in a Low Income Country. Archives of Women's Mental Health, 9, 197-202. http://dx.doi.org/10.1007/s00737-006-0129-9

[22] Lee, D.T., Yip, A.S., Leung, T.Y. and Chung, T.K. (2000) Identifying Women at Risk of Postnatal Depression: Prospective Longitudinal Study. Hong Kong Medical Journal, 6, 349-354. 